\author{
Series A
}

\author{
I. MATHEMATICA
}

311

\title{
ÜBER MEROMORPHE FUNKTIONEN AUF ENDLICH VIELBLÄTTRIGEN RIEMANNSCHEN FLÄCHEN III
}

VON

LAURI MYRBERG 


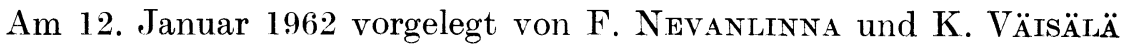




\section{Über meromorphe Funktionen auf endlich vielblättrigen Riemannschen Flächen III}

1. In den zwei ersten Teilen unserer Arbeit [2], [3] sind zwei Klassen von meromorphen Funktionen (bzw. Kovarianten) betrachtet worden:

Der Körper $f_{0}$ derjenigen auf einer nullberandeten Riemannschen Fläche $F$ meromorphen Funktionen, die alle komplexen Werte höchstens $n$-mal annehmen;

Der lineare Raum $\Phi_{0}$ der Kovarianten $\varphi=f \alpha$ mit $f \in f_{0}$ und

$$
\int_{F-G} \int_{G}|\alpha(z)|^{2} d \sigma<\infty \quad(G \text { kompakt }) .
$$

Im vorliegenden dritten Teil werden einige Unterklassen von $f_{0}$ und $\Phi_{0}$ untersucht.

2. Es sei $f(z)$ eine auf einer nullberandeten Riemannschen Fläche $F$ meromorphe Funktion, die ausserhalb einer kompakten Teilfläche $G_{0}$ den Ungleichungen

$$
0<m \leqq|f(z)| \leqq M<\infty
$$

genügt. Dann ist die Funktion $u=\log |f|$ auf $F$ eindeutig und bis auf endlich viele Singularitäten harmonisch; ferner gilt in $F-G_{0}$

$$
\log m \leqq u \leqq \log M
$$

woraus folgt (s. [4], S. 325), dass

$$
\int_{F-G_{0}} \int_{\log }|\operatorname{grad} u|^{2} d \sigma=\iint_{F-G_{0}}\left|\frac{d \log f}{d z}\right|^{2} d \sigma<\infty .
$$

3. Die Kovariante $\alpha=\frac{d \log f}{d z}$ erfüllt somit die Bedingung

$$
\iint_{F-G_{0}}|\alpha|^{2} d \sigma<\infty \quad\left(G_{0} \text { kompakt }\right) .
$$

Ferner besitzt $\alpha$ die folgenden Eigenschaften: 
$2^{\circ}$ Die Perioden $\int \alpha d z$ sind für alle Zyklen $\gamma$ rein imaginär und von der Form $2 \pi i n \stackrel{\gamma}{(n}$ eine ganze Zahl).

$3^{\circ}$ Wenn der Zyklus $d$ die Fläche $F$ in zwei Teile zerlegt: $F=F_{1} \cup F_{2}$, so dass $G_{0} \subset F_{1}$, so ist

$$
\int_{d} \alpha d z=0 .
$$

Die Richtigkeit der Behauptung $3^{\circ}$ folgt daraus, dass $\iint_{F_{2}}|\alpha|^{2} d \sigma<\infty$ und das harmonische Mass des idealen Randteiles von $F_{2}$ gleich Null ist ([4], S. 328).

$4^{\circ}$ Ist $z=z_{0}$ ein $k$-facher Pol (bzw. eine $k$-fache Nullstelle) von $f$, so besitzt $\alpha$ in $z=z_{0}$ einen einfachen Pol mit dem Residuum $k$ (bzw. $-k$ ).

$5^{\circ}$ Die Funktion $f$ nimmt die Werte 0 und $\infty$ gleich oft an; denn es gilt

$$
\begin{gathered}
n(f, 0, F)-n(f, \infty, F)=n\left(f, 0, G_{0}\right)-n\left(f, \infty, G_{0}\right)= \\
\int_{\partial G_{0}} \frac{d \log f}{d z} d z=\int_{\partial G_{0}} \alpha d z=0 .
\end{gathered}
$$

Die Anzahl der Nullstellen, $n(f, 0, F)$, ist $=n$, d.h. gleich der normalen Anzahl der $a$-Stellen.

$6^{\circ}$ Es gilt

$$
\int_{\gamma} \alpha d z=0
$$

für jeden Zyklus $\gamma$, der ausserhalb einer gewissen kompakten Teilfläche $G$ liegt.

Beweis von $6^{\circ}$. Es sei $F_{w}$ diejenige $n$-blättrige Überlagerungsfläche der $w$-Ebene, auf welche die Funktion $w=f(z)$ die Fläche $F$ konform abbildet, und $E$ die Menge der komplexen Werte, die $f$ auf $F$ weniger als $n$-mal annimmt. Die Kapazität von $E$ ist gleich Null (2, Satz 3.5). Weil die Punkte $w=0$ und $w=\propto$ nach $5^{\circ}$ nicht zu $E$ gehören, können wir ein Gebiet $G_{w}$ der $w$-Ebene finden, das die Punkte 0 und $\infty$ enthält und die Menge $E$ auslässt. Die Teilfläche $G$ von $F_{w}$, deren Spurpunktmenge in der $w$-Ebene die Menge $G_{w}$ ist, ist auf $F_{w}$ kompakt und erfüllt die Bedingung $6^{\circ}$. Es sei nämlich $\gamma$ ein Zyklus, der in $F_{w}-G$ liegt, und $\gamma$ seine Spur, wobei diejenigen Punkte, auf welchen $k$ Punkte von $\gamma$ liegen, $k$-fach mitgenommen werden. Dann gilt

$$
\int_{\gamma} \alpha d z=\int_{\gamma_{w}} d \log w=0,
$$

weil die Kurve $\gamma_{w}$ ausserhalb des Gebietes $G_{w}$ liegt. 
4. Umgekehrt, wenn eine meromorphe Kovariante $\alpha$ die Bedingungen $1^{\circ}$ und $2^{\circ}$ erfüllt, so ist

$$
f(z)=e^{\int \alpha d z}
$$

eine eindeutige meromorphe Funktion, die der Bedingung (1) genügt. Hieraus folgt aber ferner, dass $\alpha$ auch die Eigenschaften $3^{\circ}-6^{\circ}$ besitzt.

Alle Kovarianten, die die Bedingungen $1^{\circ}-6^{\circ}$ erfüllen, bilden einen linearen Raum $L$, dessen Multiplikatoren ganze rationale Zahlen sind. Der Raum $L$ ist ein Unterraum von $\Phi_{0}$.

Die Funktionen $f=e^{\int \alpha d z}$ mit $\alpha \in L$ bilden eine multiplikative Gruppe $\Gamma$, die eine Untergruppe von $f_{0}$ ist.

5. Es wird noch eine Produktdarstellung für die Funktionen der Gruppe $\Gamma$ mit Hilfe von gewissen Kovarianten des Raumes $\Phi_{0}$ gegeben. Es sei $\alpha(z, a, b)$ eine meromorphe Kovariante mit folgenden Eigenschaften:

a) Die einzigen Singularitäten von $\alpha(z, a, b)$ sind einfache Pole mit den Residuen 1 und -1 in den Punkten $a$ und $b$.

b)

$$
\int_{F-G} \int_{G}|\alpha(z, a, b)|^{2} d \sigma<\infty \quad(G \text { kompakt }) .
$$

c) Die Perioden $\int \alpha(z, a, b) d z$ auf den Zyklen $\gamma$ sind rein imaginär.

Es sei dann $f(z)^{\gamma}$ eine meromorphe Funktion der Gruppe $\Gamma$ mit den Nullstellen $b_{1}, \ldots, b_{n} \quad(k$-fache Stellen $k$-mal mitgenommen $)$ und Polen $a_{1}, \ldots, a_{n}$. Dann ist die Differenz

$$
\varphi(z)=\frac{d \log f}{d z}-\sum_{i=1}^{n} \alpha\left(z, a_{i}, b_{i}\right)
$$

eine auf $F$ analytische Kovariante, für welche

$$
\iint_{F} \varphi(z)^{2} d \sigma<\infty
$$

gilt und für welche alle Perioden $\int \psi d z$ rein imaginär sind. Weil die Fläche $F$ nullberandet ist, folgt hieraus ${ }^{\gamma} \phi \equiv 0$ oder

$$
\begin{gathered}
\frac{d \log f}{d z}=\sum_{i=1}^{n} \alpha\left(z, a_{i}, b_{i}\right), \\
f(z)=C \prod_{i=1}^{n} e^{\int a\left(z, a_{i}, b_{i}\right) d z} \quad(C \text { Konstante }) .
\end{gathered}
$$

Dies ist die gesuchte Produktdarstellung. Die Faktoren des Produktes sind i.A. nicht eindeutig, sondern sie sind multiplikative Funktionen, die beim 
Umlaufen eines Zyklus mit einem Faktor $e^{i \omega}$ ( $\omega$ reell) multipliziert werden.

6. Es sei $f(z)$ eine beliebige meromorphe Funktion des Körpers $f_{0}$, die somit alle komplexen Werte $n$-mal annimmt, höchstens von einer Menge $E$ von Kapazität Null abgesehen. Ferner seien $a$ und $b$ zwei komplexe Zahlen, die nicht zu $E$ gehören. Dann gibt es zwei positive reelle Konstanten, $m$ und $M$, und eine kompakte Teilfläche $G_{0}$ von $F$ derart, dass in $F-G_{0}$

$$
m \leqq\left|\frac{f(z)-a}{f(z)-b}\right| \leqq M
$$

gilt. Dann ist aber $f_{1}(z)=\frac{f(z)-a}{f(z)-b}$ eine Funktion der Gruppe $\Gamma$, woraus folgt, dass jede Funktion des Körpers $f_{0}$ in der Form

$$
f(z)=\frac{A f_{1}(z)+B}{C f_{1}(z)+D} \quad(A, B, C, D \text { Konstanten })
$$

dargestellt werden kann, wo $f_{1}(z) \in \Gamma$. Umgekehrt, jede Funktion von der Form (3) mit $f_{1} \in \Gamma$ gehört zu $f_{0}$.

Zusammenfassend haben wir den folgenden

Satz: Die meromorphen Funktionen, die auf einer nullberandeten Riemannschen Fläche $F$ ausserhalb einer kompakten Teilfläche $G_{0}$ den Ungleichungen (1) genügen, bilden eine multiplikative Untergruppe $\Gamma$ des Körpers $f_{0}$.

Die auf $F$ meromorphen Kovarianten mit den Eigenschaften $1^{\circ}$ und $2^{\circ}$ bilden einen linearen Raum $L$, dessen Multiplikatoren ganze rationale Zahlen sind. $L$ ist ein Unterraum des Raumes $\Phi_{0}$.

Aus $f \in \Gamma$ folgt $\alpha=\frac{d \log f}{d z} \in L$, und umgekehrt: Aus $\alpha \in L$ folgt $f=e^{\int \alpha d z} \in \Gamma$.

Jede Funktion $f$ von $I$ und die zugehörige Kovariante $\alpha$ haben die Eigenschaften $1^{\circ}-6^{\circ}$. Für $f$ gilt die Produktdarstellung (2).

Die Menge der Funktionen von der Form $\frac{A f+B}{C f+D}(f \in \Gamma ; A, B, C, D$ komplexe Zahlen) ist identisch mit dem Körper $f_{0}$. 


\section{Literatur}

[1] Ahlfors, L. V. - Sario, L.: Riemann Surfaces. - Princeton Univ. Press (1960).

[2] Mrrberg, L.: Über meromorphe Funktionen auf endlich vielblättrigen Riemannschen Flächen. - Ann. Acad. Sci. Fennicae, A I 286 (1960).

[3] -»- Über meromorphe Funktionen auf endlich vielblättrigen Riemannschen Flächen II. - Ibid. A I 301 (1961).

[4] Nevanlinna, R.: Uniformisierung. - Springer (1953). 